\title{
Implementación de una política social activa en el desarrollo sostenible del medio rural castellano-manchego
}

\section{Implementation of an active social policy for the sustainable development of rural areas in Castilla-La Mancha}

DOI: $10.46932 / \mathrm{sfjdv} 2 \mathrm{n} 2-139$

Received in: March 1st, 2021

Accepted in: May 30th, 2021

\section{Juan Andrés Buedo García}

Doctor en Ciencias Políticas y Sociología. Licenciado en Filosofía y Letras (Sección de Geografía).

Funcionario en excedencia del Cuerpo Superior de Técnicos de la Administración de la Seguridad

Social. Funcionario jubilado del Cuerpo Superior de la Junta de Comunidades de Castilla-La Mancha.

Profesor Asociado de la UCLM, Profesor-Tutor de la UNED hasta la jubilación.

Colegiado jubilado del Colegio Profesional de Ciencias Políticas

y Sociología de Castilla-La Mancha (España).

E-mail: jabuedo@telefonica.net

\section{RESUMEN}

La Comunidad Autónoma de Castilla-La Mancha es una de las más afectadas por el despoblamiento en las zonas rurales de la que eufemísticamente se ha denominado "España vaciada". Una estructura en la que juegan un papel esencial los desajustes entre población, riqueza y empleo. Engloba una problemática de largo alcance que pasa por la inevitable atención de asuntos prioritarios de carácter social; o por mejor decir, necesitan una específica "política social en favor del cambio demográfico". La puesta en funcionamiento de esta pasa por la aplicación efectiva de un desarrollo sostenible, creado ex profeso para revertir la situación, e implementado metódicamente con el objetivo principal de poner freno a la amenaza de desaparición a la que se enfrentan muchos municipios de la región. Algo que conlleva proponer e impulsar medidas para llenarlos de vida y garantizar su futuro. Con este fin se ha elaborado el documento aquí presentado, que no pasa por alto la utilización de las herramientas e instrumentos ofrecidos desde la economía circular, asumiendo también la divulgación de las determinaciones a las que llegó el II Congreso Nacional de Despoblación en el medio rural, iniciativa impulsada por la FEMP y la Diputación Provincial de Huesca.

Palabras clave: Despoblamiento, España vaciada, Cambio demográfico, Desarrollo Sostenible, Economía Circular- Política Social.

\begin{abstract}
He Autonomous Community of Castilla-La Mancha is one of the most affected by depopulation in rural areas of which euphemistically has been called "Spain emptied." A structure in which the imbalances between population, wealth and employment play an essential role. It encompasses a long-range problem that goes through the inevitable attention of priority social issues; Or rather, they need a specific "social policy in favor of demographic change." The implementation of this goes through the effective application of sustainable development, created specifically to reverse the situation, and implemented methodically with the main objective of curbing the threat of disappearance faced by many municipalities in the region. Something that involves proposing and promoting measures to fill them with life and guarantee their future. To this end, the document presented here has been prepared, which does not overlook the use of the tools and instruments offered from the circular economy, also assuming the disclosure of the
\end{abstract}


determinations reached by the II National Depopulation Congress in rural areas, initiative promoted by the FEMP and the Provincial Council of Huesca.

Keywords: Depopulation - Spain emptied - Demographic change - Sustainable Development - Circular Economy - Social Policy.

\section{INTRODUCCIÓN.}

La población censada en municipios rurales en Castilla-La Mancha descendió un 13,3 por ciento entre los años 2000 y 2018, mientras que los 907.997 habitantes de estas zonas rurales representan el 45 por ciento de toda la población castellano-manchega. Así nos lo muestra el Informe Anual de Indicadores 2018, elaborado por la Subdirección General de Análisis, Coordinación y Estadística del MAPA. En la sección reservada al análisis demográfico del medio rural se describe de manera cuantitativa esa evolución, y aclara que, con el fin de delimitar geográficamente al medio rural, se adopta la definición establecida en la Ley 45/2007 de Desarrollo Sostenible del Medio Rural, mediante la cual el medio rural sería "el espacio geográfico formado por la agregación de municipios que posean una población inferior a 30.000 habitantes y una densidad inferior a los 100 habitantes por $\mathrm{km}^{2}$ ". Junto a la definición de medio rural, se incluye la de municipio rural de pequeño tamaño: “aquel con población residente inferior a 5.000 habitantes y que esté integrado en el medio rural”. En el Gráfico 1 se presenta su evolución para el conjunto de las distintas comunidades autónomas.

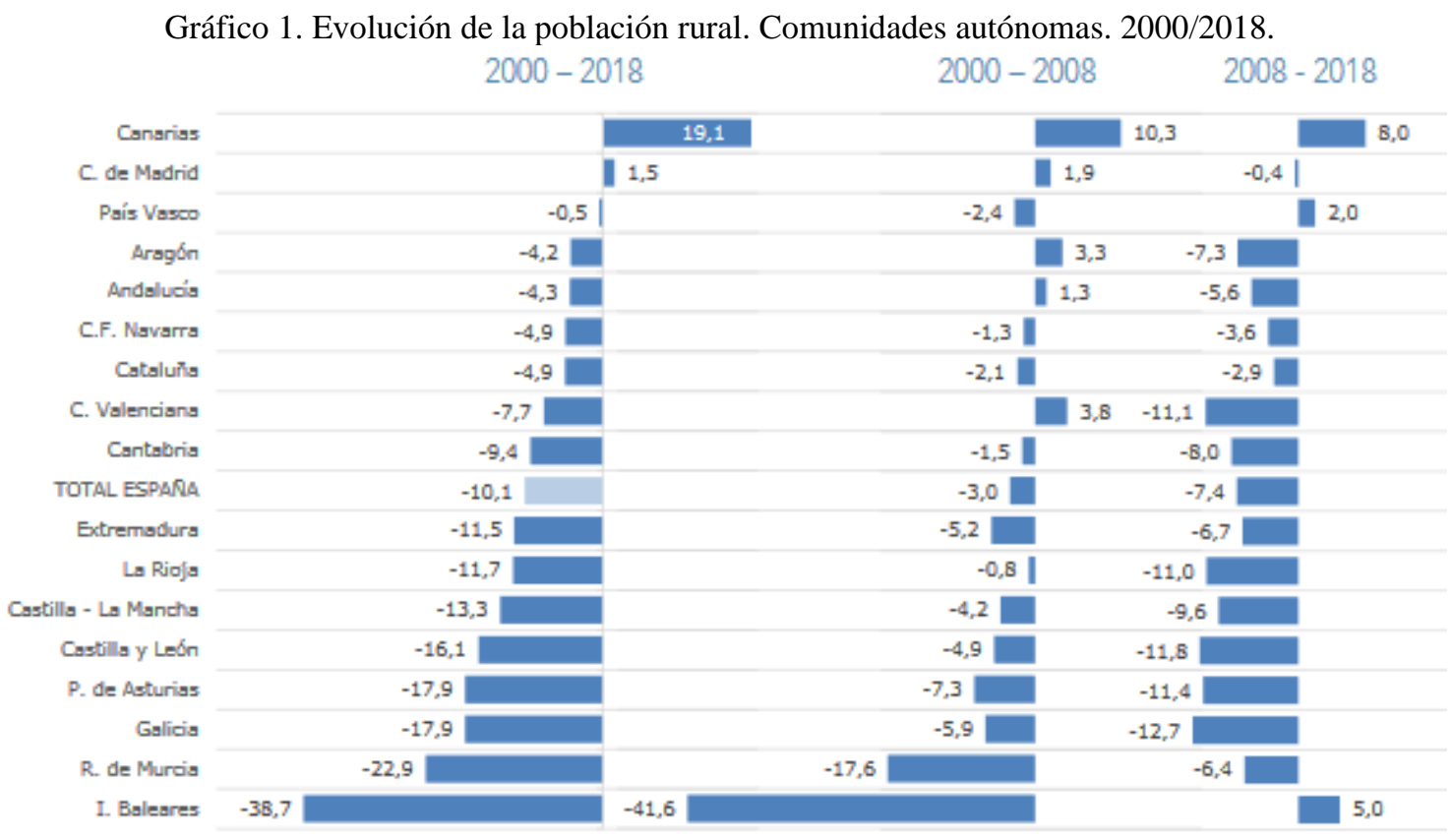

Fuente: Informe Anual de Indicadores 2018, MAPA (2019), pg. 15. 
Esta situación por si sola genera la exigencia de un conocimiento fidedigno, con la subsiguiente mejora de una intervención social metódica en la región castellano-manchega. Abarca esta ordenación a un viable sector de potencialidades e iniciativas que son ahora mismo imprescindibles para generar un nuevo emprendimiento socioeconómico en este territorio, capaz de abrir nuevos rumbos a un proyecto social preparado para terminar con el problema más adverso y contraproducente padecido por estas tierras en la actualidad: el de su despoblación y el envejecimiento de su gente, con el agregado de inconvenientes que se adosan a esta cuestión.

Son todo un cúmulo de incertidumbres, inseguridades, enigmas y obstáculos que, sobre todo, se halla necesitado de la inaplazable puesta en práctica de lo que ya se conoce como "políticas activas" contra el despoblamiento. Con este sentido entra en juego la factibilidad de crear una serie de órganos técnico-administrativos especializados en esta dirección, concebidos desde unos ángulos explícitos y acordes con la equidad social que merecen todas las personas que habitan el conjunto de la Comunidad de Castilla-La Mancha, directamente entroncadas con las surgidas en la "España vacía". El Mapa 1 muestra descriptivamente que la población se concentra en las grandes ciudades de la Comunidad. En los 36 municipios con más de 10000 habitantes reside el 55,6\% de la población, mientras que sólo reside el 7,9\% en municipios de menos de 1000 habitantes. Con la limitación añadida de un crecimiento negativo de la población en sus zonas rurales, afectadas por el fenómeno específico de la España del interior; es decir, las localidades que más población pierden en términos relativos desde 1996 están en las provincias de Guadalajara, Cuenca, Palencia y Soria.

Mapa1. Crecimiento de la Población por Municipios en Castilla-La Mancha (1998-2008) 


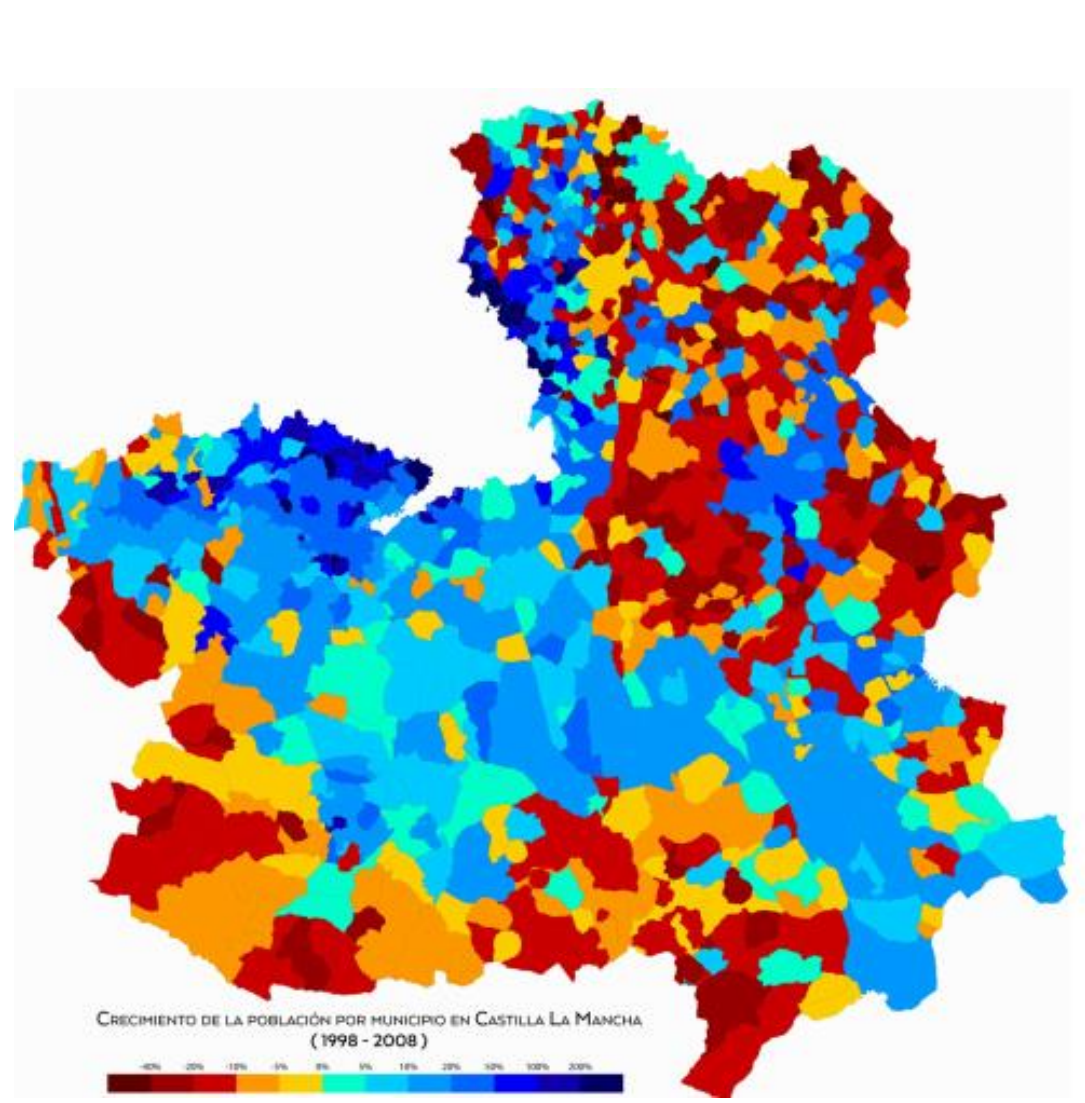

Fuente: Wikipedia. Castilla-La Mancha. Castilla-La-Mancha-crecimiento-1998-2008.png (Imagen PNG, 2000). Las políticas públicas de intervención demográfica pasan por:

$\checkmark$ El compromiso con la sociedad

$\checkmark$ La experiencia y la solidez en el campo de la acción social

$\checkmark$ La calidad y la innovación en el trabajo

$\checkmark$ La competencia profesional

$\checkmark$ La alta cualificación teórico-técnica de los equipos de trabajo

$\checkmark$ La vinculación con entidades e instituciones sociales.

La estrategia de esa política multidisciplinar, a tenor de la experiencia e indagaciones salidas del PLANCIMARCU ${ }^{1}$ pasa por la simultánea creación a nivel autonómico de la Agencia Social del Cambio Demográfico de Castilla-La Mancha. En ella debería crearse y organizar el Observatorio Demográfico de Castilla-La Mancha. Toda una estructura administrativa apta para sincronizar un conjunto de medidas reales, efectivas y rápidas que impliquen a todas las administraciones públicas, y, de manera coordinada desde la Junta de Comunidades de Castilla-La Mancha (JCCM), diseñe y ponga en práctica un proyecto multidisciplinar en el que tengan voz y voto primordial quienes conocen de primera mano este problema, los pueblos, las ciudades y las diputaciones de esta comunidad autónoma. Se estima que hay que actuar,

\footnotetext{
${ }^{1}$ El Proyecto I+D+i PLANCIMARCU, acrónimo de la investigación correspondiente, consiguió el primer premio de Ayudas a la Investigación en 2019 de la Diputación Provincial de Cuenca, y fue dirigido por el doctor Juan A. Buedo. Acabado en septiembre de ese año, el Plan de City Marketing de Cuenca constituye una estrategia de largo plazo que cuente con una institucionalidad capaz de convocar y motivar a los distintos actores públicos y privados de la ciudad para el desarrollo y materialización de proyectos, junto a nuevos desafíos comunales.
} 
pasar de las palabras a los hechos, con providencias concretas que ayuden a fijar población, a crear condiciones para el empleo y para hacer atractiva la vida en los pueblos del conjunto de la región.

La propia JCCM, a partir de la idea contenida en el anteproyecto presentado por Cuenca Abstracta en septiembre de 2019 para la creación del "Centro de Estudios para el Rejuvenecimiento de Cuenca", se ha mentalizado de la urgencia de alcanzar un Pacto contra la Despoblación en Castilla-La Mancha. Así lo hizo público el Ejecutivo de esta comunidad autónoma el 20 de enero de 2020, al insistir en la necesidad de abordar cambios en la financiación y la fiscalidad para garantizar los servicios que prestan los pequeños municipios de las zonas rurales y que, hoy por hoy, tienen "dificultades financieras" ya que toda la organización de esos servicios conlleva un "sobrecoste importante" para los ayuntamientos, más allá de las dificultades técnicas por su escasa capacidad técnica de la que disponen.

\section{LA AGENCIA SOCIAL DEL CAMBIO DEMOGRÁFICO DE CASTILLA-LA MANCHA}

El vicepresidente de la JCCM durante su declaración a los medios de comunicación en esa última fecha afirmó que tras la ronda de contactos con los agentes económicos y sociales de la región, a los que se les ha trasladado el borrador del Pacto contra la Despoblación, su intención estaba en que en el mes de febrero puedan dar por finalizado este proceso y firmar públicamente este pacto. Rubricado éste se tiene previsto poner en marcha grupos de trabajo para establecer qué medidas deben desarrollarse para frenar la pérdida demográfica, y que puedan articularse además en la futura Ley de Desarrollo Rural Territorial y contra el Despoblamiento en Castilla-La Mancha. Una norma de carácter general que ya ha iniciado su fase de consultas previas, y que se espera que pueda estar aprobada para el año 2021. A partir de ese momento se iniciará "toda una serie de mesas de trabajo donde queremos que participen instituciones y entidades para elaborar entre todos la Estrategia de Despoblación en Castilla-La Mancha”, aseveró la autoridad referida.

Ahora bien, tanto en la estrategia como en la legislación, al igual que en la implementación de su desarrollo posterior, resulta ineluctable concretar cómo deben articularse todas esas realidades y soluciones recogidas en el resumen de conclusiones agrupadas por las diversas mesas de trabajo. Han de conducir estas últimas a la puesta en práctica del conjunto de determinaciones a las que se llegó en el II Congreso Nacional DMR. Entre las conclusiones de este evento figuran como las más destacadas la necesidad de una legislación específica para el medio rural, la urgencia de que la despoblación se incluya no sólo en la agenda política española sino también en la económica, la necesidad de una fiscalidad que discrimine positivamente al medio rural, el fomento del arraigo en el medio rural, la importancia de que los fondos de la Unión Europea contemplen la despoblación, para lo que es necesario realizar medidas de lobby, o la importancia del papel de la Administración Local (ayuntamientos y diputaciones provinciales) 
en la lucha contra la despoblación. Para coordinar convenientemente este trabajo tan ingente, el “Comisionado para el Reto Demográfico de Castilla-La Mancha” debería poner en marcha la Agencia Social del Cambio Demográfico de esta Comunidad autónoma,

Esta agencia, de régimen jurídico mixto (público/privado), profesionalmente transversal y con un cometido encarado -desde la diversidad de intervención de la 'demografía aplicada'- a la prestación general de servicios englobados dentro de las grandes áreas que esta desarrolla (demografía histórica, fecundidad y familia, educación y trabajo, salud y envejecimiento, migraciones, movilidad y vivienda, etc.). Unas ayudas y unos beneficios dispuestos para atender tres grupos de demandas primordiales:

$>$ El desarrollo de indicadores y de nuevas metodologías de análisis, así como la generación y el tratamiento de fuentes de información diversas como encuestas, registros de población u otras fuentes administrativas de datos.

$>$ La realización de estudios sobre las transformaciones sociodemográficas recientes, tanto de la dinámica demográfica como de otros fenómenos sociales asociados a las poblaciones humanas susceptibles de plantear nuevos retos de gestión, como los cambios en las formas de convivencia o en la participación en el mercado de trabajo o el envejecimiento demográfico.

$>$ Responder a las necesidades de las empresas privadas y administraciones públicas de estimaciones y proyecciones de población (actividad, educación, hogares u otras características) a diferentes escalas territoriales y horizontes temporales, con el objetivo anticiparse al impacto que ejercen los cambios de las dinámicas poblacionales y de las características sociodemográficas de las poblaciones en la demanda de bienes y servicios.

\section{POLÍTICA SOCIAL SOSTENIBLE}

Se encuentra en estos momentos Castilla-La Mancha frente al desafío de perfilar políticas públicas eficaces que ofrezcan una vida digna a todos los ciudadanos, en consonancia con la Agenda 2030 para el Desarrollo Sostenible. Y justamente una buena política de población es fundamental para ello, pues, como han destacado ante la propia ONU numerosos mandatarios, "conocer la estructura de la población es indispensable para el diseño de las políticas públicas". Esto supone como primer quehacer pendiente la elaboración de una gran Guía para implementar los Retos Demográficos de Castilla-La Mancha. Y el encargo podría encomendarse precisamente a la comentada Agencia Social para el Cambio Demográfico.

Por tanto si, como está ampliamente demostrado, un aspecto ineludible a atender en la planificación y la gestión local es contar con información adecuada para la toma de decisiones, la confección de esa guía examinará el conveniente establecimiento de la "Red Intercomarcal de 
Observatorios Sociodemográficos", instalados en 5 poblaciones (una en cada provincia) y bajo la coordinación del Observatorio Sociodemográfico de Castilla-La Mancha, con sede en la reiterada Agencia. La información demográfica salida de ellos y su manejo inmediato pueden incidir en la ya demostrada evidencia de la importancia que poseen los indicadores demográficos bien elaborados y, con todo esto, se constatará cómo la estructura de la población revela los verdaderos destinatarios de las políticas a ejecutar. Podrá demostrarse a través de la recolección de un alto bloque de datos por medio de una serie de "entrevistas semi-estructuradas" a los actores institucionales responsables.

Las acciones derivadas de este proceso son de una enorme complejidad, dado que las iniciativas políticas en materia demográfica deben explicitar el diagnóstico de los problemas específicos que van a afrontar, los objetivos a los que pretenden aproximarse y los principios que inspiran dichas normas, junto a las pautas dispuestas para tratar de resolver los múltiples dilemas alrededor de estos temas.

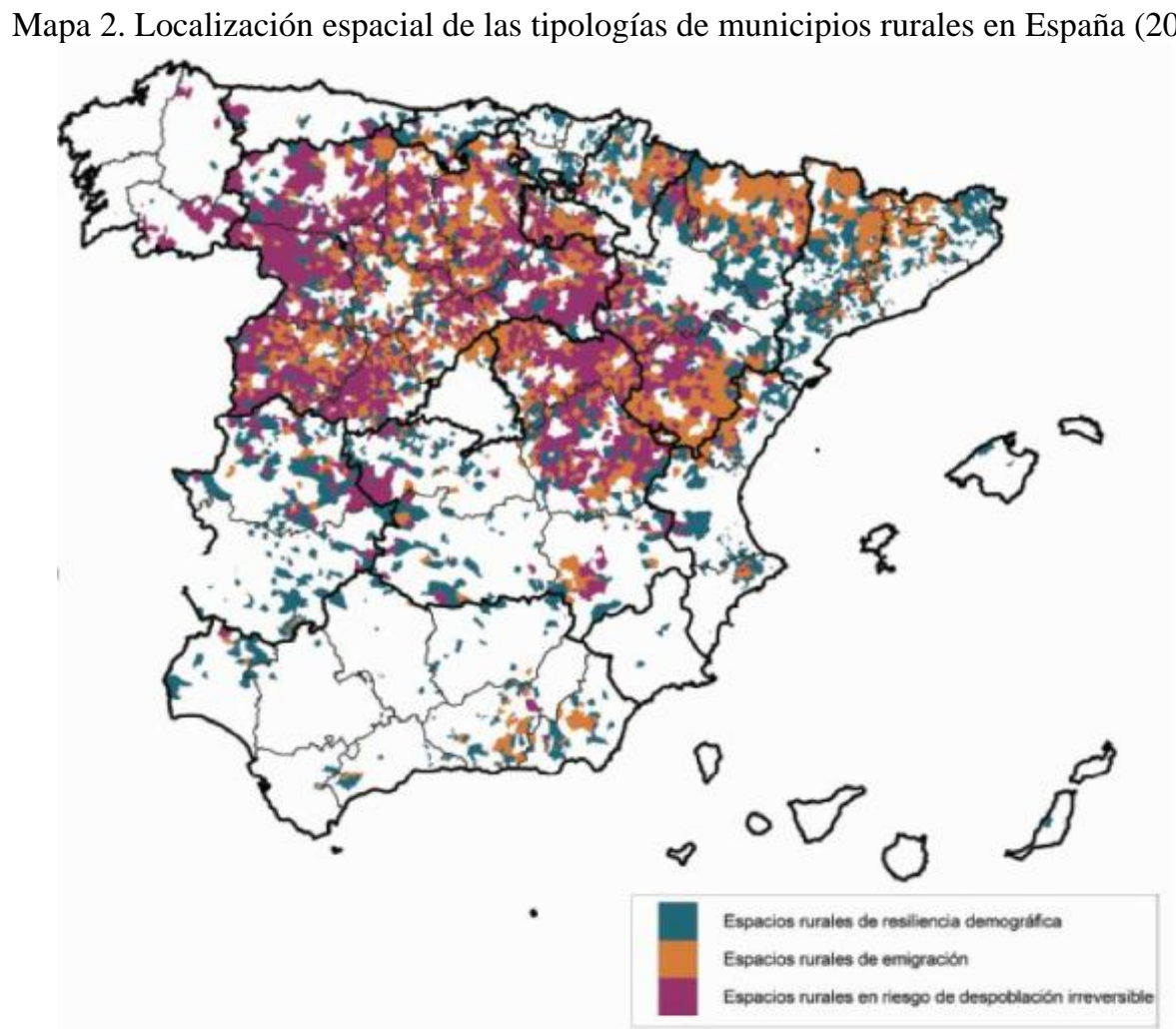

Fuente: Recaño, J., "La sostenibilidad demográfica de la España vacía”. (2017)

Podemos deducir de dichas premisas que los sucesos que condensan las estadísticas demográficas son el reflejo de actuaciones personales y colectivas sumamente complejas. Por lo que interpretarlos requiere combinar disciplinas distintas, mantener una aproximación integradora y prescindir de los prejuicios; una cuestión que, sobre todo al legislar sobre ellos, exige además tener muy en cuenta las preferencias de los ciudadanos. Se trata, en definitiva, de analizar cómo a partir de las variables demográficas se puede interpretar el comportamiento social y económico de los individuos. También, bajo 
este prisma se puede mostrar la importancia de la interconexión de las variables demográficas con otras dimensiones sociales y cómo éstas intervienen en las actuaciones de la población.

Es un vínculo combinado que enlaza con el contenido de dos de los mejores documentos que se han redactado en España sobre el diagnóstico, las respuestas y las acciones a todo este respecto en nuestro país: “Abordar el reto demográfico, hacer frente a la despoblación [1 ]” y "Estrategia Nacional frente al Reto Demográfico. Directrices generales”. Ambos contienen grandes aportaciones para el reequilibrio poblacional de cualquier territorio español y su consiguiente garantía de sostenibilidad.

Este aval nos retorna al argumento expresado en el Manifiesto de la AGE, cuando observa que el problema de la despoblación es un problema de Estado, por supuesto de todas las administraciones, pero también del conjunto de la ciudadanía, y abordarlo debe ser una política transversal, aunque adecuada a la heterogeneidad de los problemas y de los territorios. No es una cuestión que afecte única y exclusivamente a quienes viven en territorios despoblados, sino que es un problema gravísimo para el conjunto de la sociedad. Es imprescindible que las sociedades urbanas hagan suya la situación del medio rural y entiendan que las consecuencias derivadas del olvido al que está sometido un porcentaje tan alto del territorio redunda negativamente en su día a día (pérdida de cultura y tradiciones, impacto ecológico y paisajístico, pérdida de oportunidades económicas, pérdida irreversible de valores patrimoniales, etc.). Es necesario romper la dicotomía, casi siempre artificial, entre lo rural y lo urbano. Ha de apostarse por poner en positivo el papel que desempeña el medio rural como complemento imprescindible al medio urbano. Esto lo entendió muy bien el Consejo de Ministros al aprobar el 29 de marzo de 2019 un Acuerdo sobre las directrices generales de la Estrategia Nacional frente al Reto Demográfico. En él se condensa un amplio trabajo transversal y unas líneas de acción genuinamente compactadas al desarrollo sostenible

Figura 1. Claves del Desarrollo Sostenible 


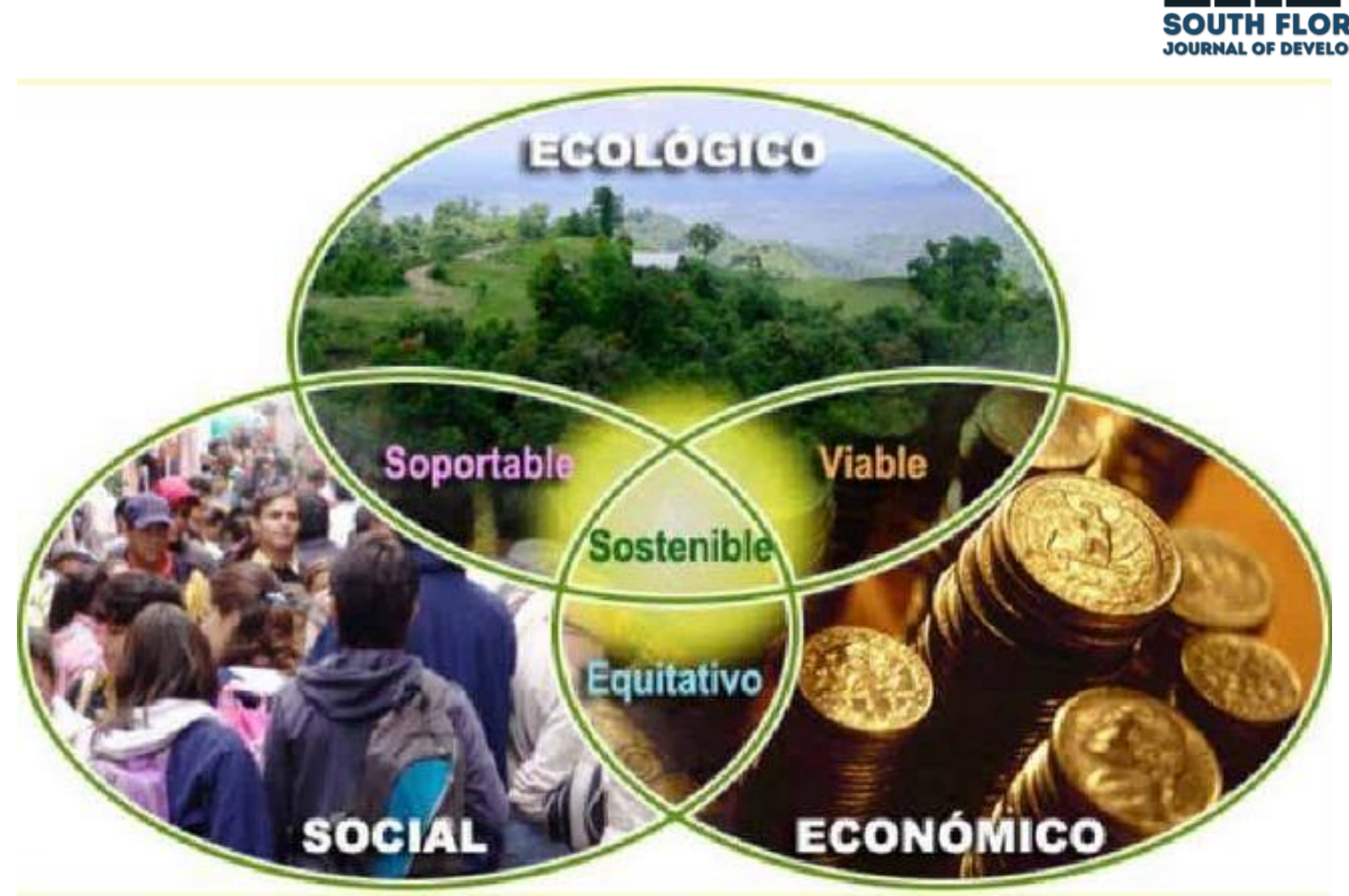

Fuente: Tálamo, G. (2018).

El Acuerdo de 29 de marzo de 2019 aclara que todos los ministerios incorporan a las directrices de la Estrategia más de 80 medidas, referidas a jóvenes, mujeres, atención a la población rural, emprendedores, turismo, implantación de la Administración en el territorio o actividad económica.

\section{Siete objetivos transversales}

- Garantizar una plena conectividad territorial, con una adecuada cobertura de internet de banda ancha y de telefonía móvil en todo el territorio, de acuerdo con la Agenda Digital Europea 2020.

- Asegurar una apropiada prestación de servicios básicos a toda la población en condiciones de equidad, adaptada a las características de cada territorio.

- Incorporar el impacto y la perspectiva demográfica en la elaboración de leyes, planes y programas de inversión, favoreciendo la redistribución territorial en favor de una mayor cohesión social.

- Avanzar en la simplificación normativa y administrativa para los pequeños municipios, con el fin de facilitar la gestión de los ayuntamientos.

- Eliminar los estereotipos y poner en valor la imagen y la reputación de los territorios más afectados por los riesgos demográficos.

- Mejorar los mecanismos para una mayor colaboración público-privada, potenciando la incorporación de los factores demográficos en la responsabilidad social del sector privado, para convertir todos los territorios, sin exclusiones, en escenarios de oportunidades. 
- Alinear las líneas de acción y los propósitos de la Estrategia con el cumplimiento de los Objetivos de Desarrollo Sostenible y de la Agenda 2030.

\section{Líneas de acción}

1.- $\underline{\text { Afrontar la despoblación }}$

- Garantizar la funcionalidad de los territorios afectados por la despoblación y la baja densidad.

- Mejorar la competitividad y facilitar el desarrollo de nuevas actividades económicas y el fomento del emprendimiento.

- Favorecer el asentamiento y la fijación de población en el medio rural.

2.- Afrontar los desequilibrios de nuestra pirámide de población

- Coordinar las acciones referidas a personas mayores, envejecimiento activo y atención a la dependencia en todo el territorio.

- Apoyar la puesta en marcha de proyectos de desarrollo socioeconómico de jóvenes, que garanticen el relevo intergeneracional.

- Facilitar el desarrollo de proyectos que garanticen la libertad de residencia efectiva de las mujeres en el territorio.

- Garantizar las condiciones que favorezcan la crianza de hijas e hijos, y que faciliten la equiparación de la tasa de natalidad al promedio de la Unión Europea.

- Asegurar la igualdad de oportunidades y la no discriminación de la infancia por ámbito de residencia.

- Trabajar, en coordinación con la UE, para canalizar una migración regular y ordenada, y su arraigo en todo el territorio.

- Facilitar la vuelta de los españoles residentes en el exterior que deseen regresar a España.

\section{3.- Gestionar los efectos de la población flotante}

- Garantizar la prestación de servicios básica para la población residente y flotante en todo el territorio.

- Asegurar el dimensionamiento de las infraestructuras y equipamientos necesarios para el desarrollo socioeconómico sostenible de las áreas con intensos flujos de población flotante.

\section{ECONOMÍA CIRCULAR EN EL MEDIO RURAL CASTELLANO-MANCHEGO}

Hay que repensar el ciclo de vida de los productos que utilizamos a diario para generar menos residuos y, en consecuencia, fomentar más ahorro energético para lo que será indispensable modificar los actuales sistemas de producción, que se apoyan en una economía lineal -basada en usar y tirar-. El nuevo 
momento ha de ser preparado en buscar un sistema alternativo en el que, gracias a la reutilización de materiales, se genere un bucle que permita que esos mismos materiales puedan volver a formar parte del sistema de fabricación y, en consecuencia, se alargue el ciclo de vida de los productos, se produzca ahorro energético y no se agoten los recursos naturales. En definitiva, la apuesta por un flujo circular de recursosproducto-recursos reciclados (descriptivamente compendiados en la Figura 2).

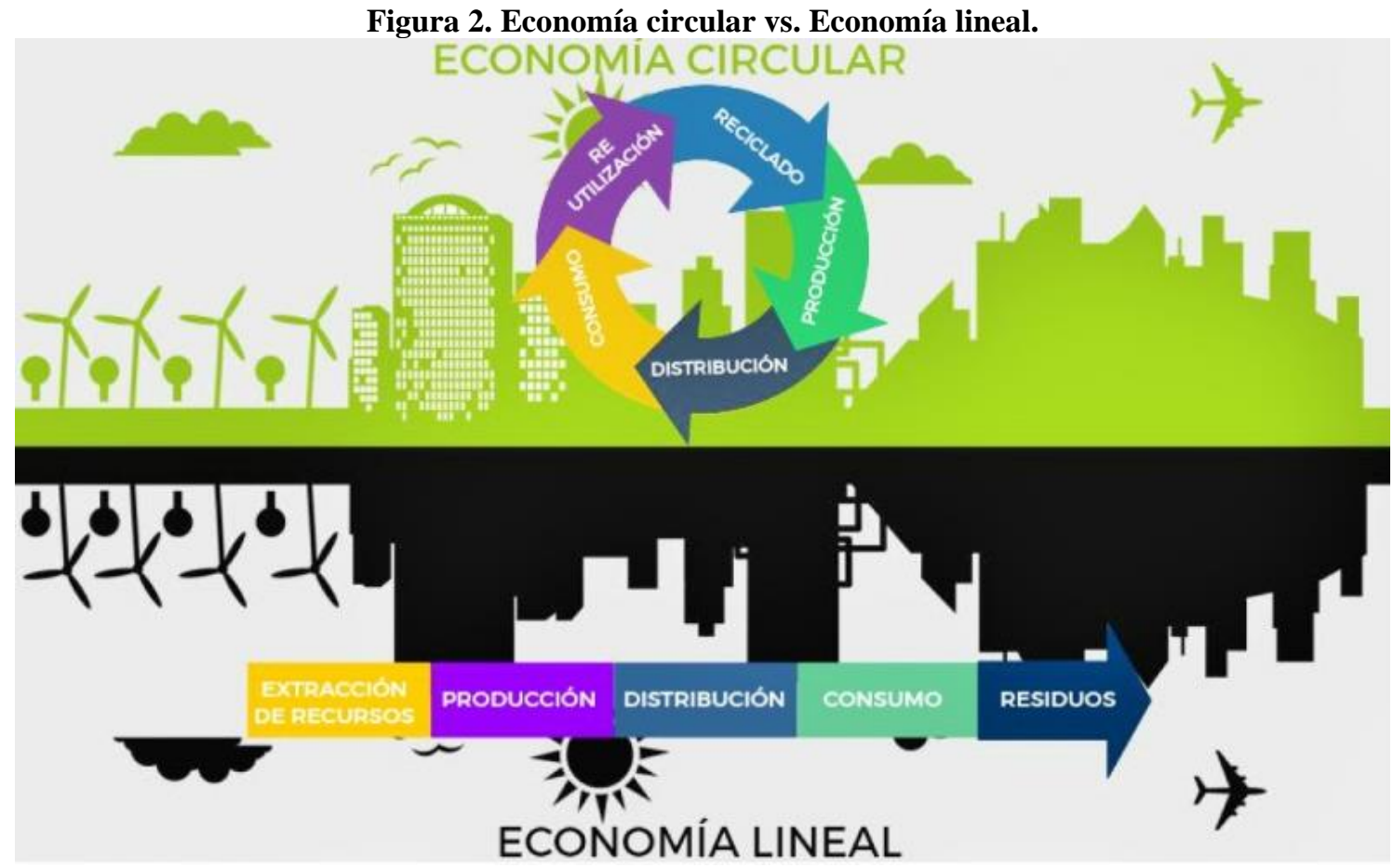

Fuente: Bartolomé, A. (2017)

Cuando hablamos de este nuevo sistema no estamos proclamando un concepto abstracto, sino que enunciamos una necesidad prioritaria de la que ya son conscientes muchos países del mundo. Así, la economía circular se hace clave para la Unión Europea, al aprobar un importante paquete de medidas ${ }^{2}$ para impulsar este modelo de economía alternativa porque -entiende- "nuestro planeta y nuestra economía no podrán sobrevivir si mantenemos el enfoque del toma, fabrica, utiliza y tira". Concretamente, el 2 de diciembre de 2015 la comisión adoptó un ambicioso nuevo paquete para impulsar la transición de Europa hacia una economía circular que impulsará la competitividad mundial, fomentará el crecimiento económico sostenible y creará nuevos puestos de trabajo. Según las estimaciones de la Comisión Europea, si se aplicara toda la normativa vigente sólo en materia de gestión de residuos se crearían más de 400.000 empleos en la Unión Europea, de los cuales 52.000 se localizarían en España.

\footnotetext{
${ }^{2}$ La Comisión Europea, en su Comunicado de prensa de fecha 2-12-2015, informa "Cerrar el círculo: la Comisión adopta un ambicioso paquete de nuevas medidas sobre la economía circular para impulsar la competitividad, crear empleo y generar crecimiento sostenible". http://europa.eu/rapid/press-release_IP-15-6203_es.htm.
} 
La Comisión Europea adoptó en 2017 un ambicioso paquete de nuevas medidas sobre la economía circular para ayudar a las empresas y los consumidores europeos en la transición a una economía más sólida y circular, donde se utilicen los recursos de modo más sostenible. Según reza en la exposición de motivos de estas medidas, las acciones propuestas contribuirán a "cerrar el círculo" de los ciclos de vida de los productos a través de un mayor reciclado y reutilización, y aportarán beneficios tanto al medio ambiente como a la economía. Estos planes extraerán el máximo valor y uso de todas las materias primas, productos y residuos, fomentando el ahorro energético y reduciendo las emisiones de gases de efecto invernadero. Las propuestas abarcan todo el ciclo de vida de los productos: de la producción y el consumo a la gestión de residuos y el mercado de materias primas secundarias. La transición recibirá ayuda financiera de los Fondos Estructurales y de Inversión Europeos (Fondos EIE), con 5.500 millones de euros para la gestión de residuos. Además, contará con 650 millones de euros procedentes de Horizonte 2020 (el programa de financiación de la investigación e innovación de la UE) y de inversiones en la economía circular a nivel nacional.

Hay diez rasgos configuradores que definen cómo debe funcionar la economía circular:

1. El residuo se convierte en recurso: es la principal característica. Todo el material biodegradable vuelve a la naturaleza y el que no es biodegradable se reutiliza.

2. El segundo uso: reintroducir en el circuito económico aquellos productos que ya no corresponden a las necesidades iniciales de los consumidores.

3. La reutilización: reusar ciertos residuos o ciertas partes de los mismos que todavía pueden funcionar para la elaboración de nuevos productos.

4. La reparación: encontrar una segunda vida a los productos estropeados.

5. El reciclaje: utilizar los materiales que se encuentran en los residuos.

6. La valorización: aprovechar energéticamente los residuos que no se pueden reciclar.

7. Economía de la funcionalidad: la economía circular propone eliminar la venta de productos en muchos casos para implantar un sistema de alquiler de bienes. Cuando el producto termina su función principal, vuelve a la empresa, que lo desmontará para reutilizar sus piezas válidas.

8. Energía de fuentes renovables: eliminación de los combustibles fósiles para producir el producto, reutilizar y reciclar.

9. La eco-concepción: considera los impactos medioambientales a lo largo del ciclo de vida de un producto y los integra desde su concepción.

10. La ecología industrial y territorial: establecimiento de un modo de organización industrial en un mismo territorio caracterizado por una gestión optimizada de los stocks y de los flujos de materiales, energía y servicios. 
El modelo económico lineal, que está llegando al límite de la capacidad física del planeta, convierte a la economía circular en una alternativa atractiva y viable también para el medio rural. Concretamente la publicación Hacia una economía circular: motivos económicos para la transición acelerada define las principales ventajas de la adopción de los fundamentos de este tipo de economía. Al trabajar fundamentalmente con procesos biológicos, es en el medio rural donde puede resultar más evidente y sencilla una primera aproximación a la economía circular llegando incluso a un impulso en su desarrollo basado en la generación de valor añadido a través de la transformación y el refinamiento de los productos rurales, más allá de la mera explotación de recursos naturales. En una sociedad donde gran parte de sus recursos provienen o dependen del rural (alimentación, agua, energía, materias primas, etc.), los procesos de transformación en el modelo productivo del campo pueden ser un impulso ejemplar para todos los sectores del sistema productivo. La implementación de políticas activas de reversión demográfica y el desarrollo sostenible en Castilla-La Mancha han de orientarse hacia la transformación y las nuevas iniciativas de los procesos circulares. En este sentido resulta interesante y aleccionador un artículo de Ignacio Belda publicado recientemente por el portal de noticias de economía circular ECO-circular.com, en especial sus epígrafes 5 y 6 , donde se responde -respectivamente- a la cuestión de por qué beneficia la economía circular al desarrollo rural y al fenómeno creciente del emprendimiento rural.

Tampoco puede obviar el gobierno de la JCCM el documento Situación y evolución de la economía circular en España, presentado en enero de 2017 por la Fundación Cotec. En este informe se recogen y analizan los indicadores disponibles, se identifican algunos de los principales actores implicados, así como casos de éxito y buenas prácticas. Se afirma en dicha referencia que la innovación es el elemento clave para lograr la transición hacia una economía circular. Luego serán necesarias nuevas tecnologías, procesos, servicios y modelos empresariales, así como el cambio integral en los patrones de comportamiento de los consumidores.

La gestión de todos estos cambios exige a las administraciones y las empresas disponer de datos e información para la toma de decisiones y la asignación de recursos. Pero en muchos casos esta información todavía no está disponible o lo está de forma fragmentada. Con ese informe, Cotec quiere contribuir a cubrir las carencias que existen en este sentido, a través de la elaboración de un mapa de situación de la economía circular en España.

La transición de la economía circular en España representa una gran oportunidad para el desarrollo económico y para la creación de nuevos yacimientos de empleo sostenible, pero para dar un impulso a este potencial innovador, como destaca Albert Vilariño ${ }^{3}$, es necesario armonizar esfuerzos y definir

\footnotetext{
${ }^{3}$ Albert Vilariño (2017): “¿Cómo avanza la economía circular en España y en Europa?”, Compromiso Empresarial, 15 de junio de 2017. Disponible en: https:/www.compromisoempresarial.com/rsc/2017/06/como-avanza-la-economia-circular-enespana-y-en-europa/
} 
estrategias de largo plazo y acciones inmediatas. En la actualidad todavía no se cuenta con una metodología específica que esté suficientemente elaborada y consensuada para el seguimiento y evaluación de los procesos de economía circular. Además, los sistemas de indicadores aún no están totalmente desarrollados, especialmente los relativos a la prevención del uso excesivo de materias primas, el ecodiseño y la ecoinnovación.

El informe de Cotec destaca una serie de mejores prácticas y realiza un análisis de políticas autonómicas que se están realizando para el impulso de la economía circular. Pero tras un rápido vistazo a ambas cosas se ve que la mayoría están dirigidas a la gestión de residuos, dejando bastante olvidadas (al igual que pasaba con los indicadores) las fases iniciales de producción y consumo. Entonces, para facilitar la transición hacia la economía circular en España, y en Castilla-La Mancha particularmente, son necesarias nuevas iniciativas políticas, empresariales y sociales centradas en el objetivo general de fomentar las capacidades endógenas, en línea con lo marcado por la Comunidad Europea. Son decisiones que en el ámbito de la región en la nos hemos centrado han de ponerse en marcha por las instituciones castellano-manchegas, con el objeto de establecer unas iniciativas que, para que sean realmente efectivas, deben circunscribirse a la hoja de ruta nacional para la economía circular ${ }^{4}$. A ésta le corresponde involucrar a todos los actores principales, tiene que contar con adecuados mecanismos de coordinación y participación transversal para cumplir con los planteamientos estratégicos de la UE, y ha de acomodarse para hacer frente a los retos principales derivados del desarrollo de políticas, de una reforma de la fiscalidad y de la necesidad de formación.

\footnotetext{
${ }^{4}$ Como dice el Informe Cotec (pg. 89): "Para aprovechar de manera sistémica las ventajas de la Economía Circular, que implica un cambio radical del sistema de producción y consumo, es necesario definir una clara hoja de ruta, que comprenda objetivos y estrategias a largo plazo, así como medidas y acciones de corto plazo, y que integre los esfuerzos a diferentes niveles (estatal, autonómico y local) comprendiendo también el rol de las ciudades y de sectores industriales específicos".
} 


\section{CONCLUSIONES}

La brevedad de la ponencia, al elucidar sus resultados y derivaciones, como se ha puesto de manifiesto en casi todos sus apartados y suscribe el mismo párrafo final, permite concentrarse en un punto cardinal que se emplaza, por encima de leyes o proyectos dilatorios, en la creación y la inmediata puesta en funcionamiento del Observatorio Sociodemográfico de Castilla-La Mancha, que se concibe como un órgano colegiado de asesoramiento, colaboración institucional y participación social en materia de demografía en el ámbito de esta Comunidad Autónoma, con el fin de agrupar, promover, organizar y coordinar la futura Red Intercomarcal de Observatorios Sociodemográficos de esta Comunidad Autónoma. De no realizar este consejo se avanza -desde este mismo momento- que la situación se haría "irrecuperable", según demuestran los datos que se han presentado aquí.

La primera tarea concebida es la pronta elaboración de un amplio 'Informe Castilla-La Mancha: perspectivas demográficas', documento que recoja una diagnosis de la situación castellano-manchega desde el punto de vista demográfico, tras cinco o seis meses de un trabajo de campo técnico, transversal y lo más completo posible, que con las herramientas adecuadas dé paso a análisis precisos.

Podría así dinamizarse, bajo una forma y encuadre análogos a la "Red Aragonesa de Desarrollo Rural”, bajo la gestión de una página Web que, con el nombre indicado (OSDCASMAN), distribuyera distintos habitáculos administrativos, con sus departamentos convenientes:

\begin{tabular}{|c|c|c|c|c|c|c|}
\hline Inicio & RIOSD & $\begin{array}{l}\text { Grupos } \\
\text { Leader }\end{array}$ & Noticias & Publicaciones & $\begin{array}{c}\text { Enlaces } \\
\text { Web }\end{array}$ & Eventos \\
\hline
\end{tabular}

Su finalidad central está en propiciar el análisis crítico y el debate a partir de la observación del estado y dinámica demográfica que permita comprender el impacto de los cambios demográficos en diferentes áreas de las comarcas y la región en su conjunto, junto a su interacción con la dinámica demográfica española y europea. Vendría a ofrecer al público en general información sobre variables demográficas (mortalidad, fecundidad y migración) que inciden directa e indirectamente en los procesos sociales que se desarrollan en Castilla-La Mancha.

Ha de ser un lugar donde se observe, analice, procese, actualice y teorice acerca de una realidad o contexto determinado. Además, constituye una herramienta útil para construir información y/o conocimiento a partir de datos estadísticos, cuantitativos y cualitativos, diagnósticos y documentos analíticos, que tienen como objeto explicar o aportar conceptos para la compresión de los fenómenos sociales que afectan a una población concreta.

Como meta principal establecería la observación y sistematización de los indicadores que reflejan la estructura y estado demográfico de Castilla-La Mancha y a la vez visibilizar y poner información al 
alcance público en general por medio de una plataforma virtual; un programa de acceso libre en donde los usuarios podrán procesar en línea la información de su interés.

Para ello vendría a establecer en su Web un Geoportal Demográfico de la Comunidad Autónoma. Un portal interactivo que permita visualizar información en forma de mapas, a nivel de región, provincia y municipio. Puede ser elaborado con unos 120 indicadores de datos sociodemográficos y de las desigualdades. Los indicadores son de estructura de la población, dinámica de población, desigualdad en pobreza y desigualdad en educación, empleo, sanidad y TICs. Y organizar las bases de datos espaciales a nivel de región, provincia y municipio.

La creación y el desarrollo normativo del Observatorio se puede hacer a través de un decreto del Gobierno de la Junta de Comunidades de Castilla-La Mancha, que apruebe la "Instrucción General de Política Demográfica y contra la Despoblación de Castilla-La Mancha”. Esta disposición administrativa debe fijar las actuaciones de aquél, que han de plasmarse en la elaboración de informes periódicos, al menos de carácter bianual, para ayudar a dirigir y adaptar las políticas públicas castellano-manchegas en materia demográfica y de lucha contra la despoblación para la atención de las necesidades propias de la Comunidad Autónoma.

Y las funciones destacadas de su intervención pueden ser las siguientes:

a) Estudiar, analizar y divulgar información y soluciones relevantes en materia demográfica y poblacional.

b) Realizar el seguimiento y la evaluación de los efectos de los objetivos, estrategias y medidas contenidas en la Instrucción General de Política Demográfica y contra la Despoblación, así como de la evolución de los indicadores contenidos en la misma.

c) Realizar el seguimiento del Programa de Gestión Territorial que deriva de la Instrucción General de Política Demográfica y contra la Despoblación.

d) Elaborar y formular propuestas que pudieran integrarse y/o caracterizar líneas estratégicas y prioridades de actuación del Gobierno de Castilla-La Mancha.

e) Realizar cuantas evaluaciones le encarguen las Administraciones Públicas en esta materia.

f) Analizar los efectos de las políticas en materia demográfica y poblacional para reforzar las primeras y evitar los errores cometidos de las mismas.

g) Coordinar y realizar guías informativas de los servicios que presta la JCCM en materia demográfica y poblacional.

h) Identificar iniciativas, experiencias, proyectos piloto, recursos y programas nacionales e internacionales en materia de dinamización demográfica y poblacional, que ayuden a incorporar casos de éxito en las políticas públicas castellano-manchegas. 
i) Elaborar una base de datos que incluya los costes y calidad de los todos servicios públicos en los asentamientos de población para evaluar las diferentes necesidades de financiación que requieren la prestación de los mismos en función de su ubicación territorial.

j) Crear un catálogo permanente de las medidas y políticas públicas vigentes con efectos directos sobre la demografía y el mantenimiento de la población en el territorio en los diferentes ámbitos territoriales administrativos. A este catálogo inicial de medidas se irán añadiendo aquellas que se vayan poniendo en práctica con la aprobación y aplicación de la Instrucción.

k) Diseñar, en colaboración con los diversos departamentos universitarios interesados, un método para evaluar la población real de los asentamientos con objeto de mejorar la gestión de los servicios municipales y posibilitar el establecimiento de fórmulas de acceso más eficaces y eficientes a los equipamientos públicos.

1) Promover foros de debate y trabajo que permitan el intercambio de conocimiento y experiencias entre las personas que habitan el territorio castellano-manchego, Administraciones Públicas y organizaciones sociales implicadas en la temática del Observatorio.

m) Apoyar técnicamente a la elaboración del Monográfico sobre Demografía y Población vigentes en Castilla-La Mancha..

n) Crear un espacio virtual para que, a través de las tecnologías de la información y comunicación (TIC), los territorios puedan aportar propuestas concretas de desarrollo y concreción de las medidas contempladas en las subsiguientes instrucciones futuras de la JCCM.

o) Valorar la eficacia de las inversiones públicas destinadas a progresar en el equilibrio demográfico y poblacional.

p) Realizar campañas de sensibilización sobre el patrimonio natural y cultural de la Comunidad Autónoma y sobre el medio rural.

q) Cualesquiera otras que se consideren relevantes en materia demográfica y poblacional y le sean encomendadas por la Consejería competente en materia de ordenación del territorio o por organismo público dependiente de la Administración de la Comunidad Autónoma.

La construcción del Atlas Sociodemográfico de Castilla-La Mancha y la sucesiva actualización periódica, sería la culminación del trabajo amparado por el OSDCASMAN. Una labor que debe acomodarse dentro de la redefinición de las políticas para el desarrollo sostenible, con el fin de cerrar las brechas y superar las contradicciones en la aplicación de la Agenda 2030 producidas en la Comunidad autónoma. El título de ese memorándum (“Transformando nuestro mundo”) señaló, como recordaba Jens Martens, Director del Global Policy Forum, que debería desencadenar cambios fundamentales en la política y la sociedad. Con esta Agenda, los gobiernos se comprometieron a cambiar de rumbo y a dejar 
las políticas “inerciales”. Sin embargo, cuatro años después de su adopción, la mayoría de los gobiernos no han logrado convertir la proclamada visión transformadora de la Agenda 2030 en políticas reales. Peor aún, las políticas de un número creciente de países se están moviendo en el sentido opuesto, socavando gravemente el espíritu y los objetivos de la Agenda 2030.Existen alternativas audaces e integrales al modo habitual de proceder que ayudarían a cambiar el rumbo hacia políticas fiscales y regulatorias más coherentes. Por tanto, es necesario adoptar un enfoque de sostenibilidad que involucre al conjunto de agencias de cada gobierno. Los jefes de Estado -en connivencia dentro de España con los regidores de las Comunidades autónomas- deben declarar prioritaria la aplicación de la Agenda 2030 y de los ODS. "Las estrategias nacionales de desarrollo sostenible no han de considerarse como una entre muchas, sino convertirse en el marco general de todas las políticas”, reiteró Martens y así entiendo que debe hacerse por parte de la JCCM.

Si bien, no está de más reconocer que la aplicación de la Agenda 2030 no es solo una cuestión de políticas mejores. La eficacia de las reformas políticas necesarias en el proceso de aplicación de la Agenda de 2030 requiere cambios holísticos en las estructuras de poder, y depende de la existencia de instituciones públicas sólidas, democráticas y transparentes a nivel nacional e internacional. El Foro Político de Alto Nivel (FPAN) de 2019 a nivel de Jefes de Estado y de Gobierno, la subsiguiente revisión del FPAN y el 75..$^{\circ}$ aniversario de la ONU en 2020 ofrecen nuevas oportunidades para fortalecer y renovar el marco institucional para el desarrollo sostenible en la ONU. Y, desconcentrada y paralelamente, debe producirse en el nivel inmediato inferior: las Comunidades autónomas de toda España; entre ellas, como no, en Castilla-La Mancha. 


\section{REFERENCIAS BIBLIOGRÁFICAS}

Asociación de Geógrafos Españoles (2018): Manifiesto Despoblación, AGE y Colegio de Geógrafos de España, Valladolid, 13 de diciembre de 2018. Disponible en: https://www.agegeografia.es/site/manifiesto-despoblacion/. Consultado el 15/02/2020 a las 17:58.

Bartolomé Sualdea, A. (2017): "Economía circular y mundo rural”, Carrión Digital, 3 de octubre de 2017. Disponible en: http://carriondigital.com/noticia/2017-10-03-economia-circular-mundo-rural-1009. Consultado el 16/02/2020 a las 11:10.

Belda Hériz, I. (2020): "Economía circular, una oportunidad para revitalizar el mundo rural”, ECOcircular.com, 28 de enero de 2020. Disponible en: https://eco-circular.com/2020/01/28/economiacircular-una-oportunidad-para-revitalizar-el-mundo-rural/. Consultado el 16/02/2020 a las 13:38.

Buedo García, J.A. (2019): "Centro de Estudios para el Rejuvenecimiento de Cuenca". Proyecto de Creación presentado por Cuenca Abstracta 2016 a la JCCM, Cuenca, septiembre 2019, 25 pgs.

Buedo García, J.A. (2019): “¿Y ahora qué? Efectos anómalos de la bomba demográfica castellanomanchega", La Vanguardia de Cuenca, 15 de agosto de 2019. Disponible en: https://jabuedo.typepad.com/la_vanguardia_de_cuenca/2019/08/y-ahora-qué-efectos-anómalos-de-labomba-demográfica-castellano-manchega. Consultado el 15/02/2020 a las 16:52.

Comisionado del Gobierno Frente al Reto Demográfico (2019): Estrategia Nacional frente al Reto Demográfico. Directrices generales, Ministerio de Política Territorial y Función Pública, Madrid.

FEMP (2019): “Conclusiones del II Congreso Nacional de Despoblación, y audios de todas las mesas de debate. \#PoblarLaDespoblación"; incluye el pdf del Listado de medidas para luchar contra la despoblación en España. Disponible en: http://www.femp.es/comunicacion/noticias/despoblacion-unaley-especifica-con-toda-su-financiacion. Consultado el 15/02/2020 a las 16:19.

Fernández, D. y Sierra, A. (2012): La biopolítica en el mundo actual: Reflexiones sobre el EFECTO FOUCAULT. Laertes S.A. de Ediciones, Barcelona.

Fernández, M. (2018): Contra la despoblación (Europa en la encrucijada). Bubok Publishing S.L., Madrid.

Franco, J. (2017): Diseño de Políticas Públicas: Una guía práctica para transformar ideas en proyectos viables. IEXE Editorial, Ciudad de Puebla (México).

Fundación Cotec (2017): Situación y evolución de la economía circular en España, Fundación COTEC para la Innovación, Madrid.

Fundación Ellen MacArthur (no consta año): Hacia una economía circular: Motivos económicos para una transición acelerada. Disponible en: https://www.ellenmacarthurfoundation.org/assets/downloads/publications/Executive_summary_SP.pdf. Consultado el 16/02/2020 a las 12:44.

Innerarity, D. (2020): Una teoría de la democracia compleja. Galaxia Gutenberg, S.L., Barcelona. 
Martens, J. (2018): "Redefinir las políticas para el desarrollo sostenible", Global Policy Watch. Observatorio de políticas globales. Disponible en: https://www.globalpolicywatch.org/esp/?p=613. Consultado el 16/02/2020 a las 16:15.

Ministerio de Agricultura, Pesca y Alimentación (2019): Informe Anual de Indicadores 2018. Subsecretaría del Ministerio de Agricultura, Pesca y Alimentación (MAPA), Subdirección General de Análisis, Coordinación y Estadística: Madrid. Disponible en: https://www.mapa.gob.es/es/ministerio/servicios/analisis-y-prospectiva/informe_anual_ indicadores2018_tcm30-513683.pdf. Consultado el 15/02/2020 a las 11:06.

Recaño, J.(2017): "La sostenibilidad demográfica de la España vacía”, Perspectives Demogràfiques, n 007, julio 2017, Centre d'Estudis Demográfics, Universitat Autònoma de Barcelona. Disponible en: http://ced.uab.es/es/difusion/butlleti-perspectives-demografiques. Consultado el 15/02/2020 a las 18:07.

Ribeiro, T. (2020): "Pattern Recognition in Scientific Social Networks: A Systematic Literature Review", South Florida Journal of Development, Miami. Disponible en: https://southfloridapublishing.com/ojs/index.php/jdev/issue/view/5.

Sachs, J.D. (2015): La era del desarrollo sostenible. Nuestro futuro está en juego: Aupemos el desarrollo sostenible a la agenda política mundial. Deusto, Editorial Grupo Planeta, Barcelona.

Saucedo, S.E. (2014): Gobierno, territorio y población: las políticas públicas en la mira. El Colegio de México, A.C., México.

Tálamo, G. (2018): “¿Desarrollo sustentable o sostenible?”, Planeta Vital, 9 de mayo de 2018. Disponible en: https://tuplanetavital.org/actualidad-planetaria/\%C2\%BFdesarrollo-sustentable-o-sostenible/. Consultado el 15/02/2020 a las 18:50.

Wikipedia. Castilla-La Mancha. Disponible en: https://es.wikipedia.org/wiki/Castilla-La_Mancha. Consultado el 15/02/2020 a las 14:06. 